These effects seem to be more remarkable in patients who took fish oil in a higher dose and for a longer duration.

\section{GW23-e0110 EFFECTS OF FISH OIL SUPPLEMENTATION ON INFLAMMATORY MARKERS IN CHRONIC HEART FAILURE: A META-ANALYSIS OF RANDOMISED CONTROLLED TRIALS}

doi:10.1136/heartjnl-2012-3029200.10

Objectives Effects of additional fish oil supplementation on systematic inflammation in patients with chronic heart failure (CHF) remain controversial. We performed a meta-analysis to evaluate effects of oral fish oil intake on circulating levels of inflammatory markers in patients with CHF.

Methods Human intervention studies evaluating fish oil supplementation in CHF patients were identified by systematic search of Medline, Embase, Cochrane's library and references cited in related reviews and studies through November 2011. Either a fixed-effect model or, in the presence of heterogeneity, a random-effect model was used to estimate the combined effects.

Results A total of seven trials were reviewed. Meta-analysis results showed circulating levels of tumour necrosis factor $\alpha(\mathrm{SMD}=$ $-0.62,95 \% \mathrm{CI}-1.08$ to $-0.16, \mathrm{p}=0.009)$, interleukin $1(\mathrm{SMD}=$ $-1.24,95 \% \mathrm{CI}-1.56$ to $-0.91, \mathrm{p}<0.001)$ and interleukin $6(\mathrm{SMD}=$ $-0.81,95 \%$ CI -1.48 to $-0.14, p=0.02$ ) were significantly reduced in fish oil group compared with placebo; however, circulating levels of high sensitivity $C$ reactive protein $(S M D=-0.02,95 \% \mathrm{CI}-0.26$ to $0.21, p=0.84)$, soluble intracellular adhesion molecular 1 $(\mathrm{SMD}=-0.19,95 \% \mathrm{CI}-0.97$ to $0.58, \mathrm{p}=0.63)$ and vascular cell adhesion molecular 1 (SMD $=-0.06,95 \% \mathrm{CI}-0.33$ to 0.21 , $\mathrm{p}=0.65)$ remained unchanged. Meta-regression and subgroup analysis indicated the differences in dose of fish oil and follow-up duration might influence the effects of fish oil on the inflammatory markers significantly. Greater reduction of these inflammatory markers might be identified in patients taken higher dose of fish oil (over $1000 \mathrm{mg} /$ day) for a longer duration (over 4 months).

Conclusions Anti-inflammation may be a possible mechanism underlying the potential therapeutic role of fish oil for patients with CHF who were already on current optimal medications. 